hin, dass Zweifel an der Anwendbarkeit des Verfahrens nach $\$ 211$ a SGB V bestehen. Zum einen dürfte es an einer „,nach diesem Gesetz gemeinsam und einheitlich zu treffenden Entscheidung" i.S.d. \$211a S. 1 SGB V fehlen (vgl. hierzu Krauskopf, in: Krauskopf, Soziale Krankenversicherung - Pflegeversicherung, SGB V, \211a, Rdnr. 3, Stand: Februar 2009). Zum anderen dürfte das in \$110 Abs. 1 S. 5 ff. SGB V bestimmte besondere Verfahren vorrangig Anwendung finden.

Schließlich bezieht sich die vom Kl. bemühte Berechtigung nach $\$ 212$ Abs. 5 S. 10 SGB V allenfalls auf die (anderen) Ersatzkassen, nicht aber auf die Landesverbände der Krankenkassen. [...]

https://doi.org/10.1007/s00350-021-5800-7

\section{Anmerkung zu Niedersächsisches OVG, Urt. v. 16.7.2020 - 13 LC 302/19 (VG Braunschweig)}

\section{Heike Thomae}

Die Entscheidung des Nds. OVG berührt eine Reihe von Rechtsfragen in Bezug auf die Kündigung von Versorgungsverträgen nach dem SGB V. Die Zulassung von Krankenhäusern zur Versorgung gesetzlich Krankenversicherter vollzieht sich im Wesentlichen durch die Krankenhausplanung der Länder. Aus dem Nebeneinander von Zulassungsrecht der GKV und staatlicher Krankenhausplanung ergeben sich zahlreiche Probleme. Das Nds. OVG befasste sich konkret mit der von den Krankenkassen ausgesprochene Kündigung des (fingierten) Versorgungsvertrages eines Plankrankenhauses, der die zuständige nds. Krankenhausplanungsbehörde widersprach, und der Klage einer einzelnen Krankenkasse hiergegen. Zu der Frage, ob überhaupt Kündigungsgründe bestanden, die in \$109 Abs. 3 S. 1 SGB V genannt sind, gelangte das Gericht nicht, da es schon auf formaler Ebene die Klage der einzelnen Krankenkasse wegen fehlender Klagebefugnis als unzulässig abwies, denn die Klagebefugnis stehe nur den Landesverbänden der Krankenkassen und der Ersatzkassen gemeinsam zu. So wie das Recht zur Kündigung des Versorgungsvertrages kraft ausdrücklicher Anordnung nach S110 Abs. 1 SGB V nur den beteiligten Landesverbänden der Krankenkassen und Ersatzkassen gemeinsam zusteht, müsse gleiches gelten für die an diese - nur gemeinsam mögliche Kündigung - anknüpfenden Rechtspositionen auf Genehmigung der Kündigung durch die zuständige Landesbehörde einschließlich des damit einhergehenden Anspruchs auf Aufhebung eines die Genehmigung versagenden Entscheides und auch auf Feststellung eines Eintritts der Genehmigungsfiktion nach $\$ 110$ Abs. 2 S. 4 SGB V. Zutreffend verweist das OVG darauf, dass für dieses Auslegungsergebnis nicht nur der offensichtlich systematische Regelungszusammenhang spricht, sondern auch Sinn und Zweck der Anordnung eines gemeinsamen Handelns, das gerade das unabgestimmte Vorgehen eines einzelnen Landesverbandes der Krankenkassen oder einer einzelnen Ersatzkasse zu verhindern und sich daraus ergebende Probleme für den Fortbestand oder die Abwicklung des einen Versorgungsvertrags zu vermeiden suchte. Auch die Zuständigkeit der Verwaltungsgerichtsbarkeit hat das Nds. OVG zutreffend bejaht. Grundsätzlich entscheiden zwar die Gerichte der Sozialgerichtsbarkeit in Angelegenheiten nach dem SGB V u. a. aufgrund der Be-

Rechtsanwältin Dr. iur. Heike Thomae,

Fachanwältin für Medizinrecht,

Quaas \& Partner Rechtsanwälte mbB,

Märkische Str. 115, 44141 Dortmund, Deutschland ziehungen zwischen Krankenhäusern und Krankenkassen einschließlich ihrer Vereinigungen und Verbände sowie aufgrund von Entscheidungen oder Verträgen der Krankenkassen oder ihrer Verbände, dies gilt jedoch nicht für Streitigkeiten, die in Angelegenheiten nach $\$ 110$ SGB V aufgrund einer Kündigung von Versorgungsverträgen entstehen. Insoweit sind die Verwaltungsgerichte zustän$\operatorname{dig}^{1}$. Der Rechtsweg zu den Verwaltungsgerichten soll divergierende Entscheidungen im Zusammenhang mit der Kündigung von Plankrankenhäusern vermeiden. Da die Kündigung mit einem Antrag auf Aufhebung oder Änderung des Feststellungsbescheides zu verbinden ist, ist es sinnvoll, etwaige Anfechtungen der Entscheidungen einheitlich vor den Verwaltungsgerichten auszutragen. Die Beendigung von Versorgungsverträgen ist - wie die vorliegende Entscheidung verdeutlicht - ein komplexes Verfahren, das mit seinen vielfältigen Rechtsproblemen in seiner Gesamtheit in den Bereich der Krankenhausplanung fällt, für die der Verwaltungsrechtsweg gemäß $₫ 40$ Abs. 1 VwGO eröffnet ist.

1) So bereits BSG, Beschl. v. 24.4.1995 - 3 BS 1/94.

\section{Genehmigung zur Durchführung und Abrechnung von sonographischen Leistungen nach GOP 33050 EBM für Facharzt für physikalische und rehabilitative Medizin}

SGB V §135 Abs. 2 S. 1 und S. 2; SGG §197a Abs. 1 S. 1 Teils. 3 i. V. mit §154 Abs. 1 VwGO; BMV-Ä §11 Abs. 1

S. 1; Ultraschall-Vereinbarung $\S 14$ Abs. 6 i. V. mit $\S 6$ Abs. 1 Buchst. c

Für die Beurteilung, ob Leistungen fachzugehörig oder fachfremd sind, ist grundsätzlich auf die aktuelle Fassung der WBO der für den Vertragsarztsitz örtlich zuständigen Kammer abzustellen (Leitsatz des Bearbeiters).

BSG, Urt. v. 15. 7.2020 - B 6 KA 19/19 R (LSG für das Saarland)

Problemstellung: Der Senat hatte 2016 in Fortführung seiner Rechtsprechung entschieden, dass die $\mathrm{KÄV}$ einem Facharzt für diagnostische Radiologie die Genehmigung für die Erbringung und Abrechnung von strahlentherapeutischen Leistungen zu Recht versagte, weil er die fachlichen Voraussetzungen nicht erfüllte und zudem die Leistungen für ihn fachfremd sind.

So eingängig dieses Urt. im Ergebnis war, haben doch Aufbau des Urt. und einzelne Formulierungen im Urt. die Frage aufgeworfen, ob der Senat an seiner Rechtsprechung festhält, dass

- die KÄV einem Vertragsarzt, wenn er die fachliche und apparative Voraussetzung für die Erbringung einer bestimmten Leistung erfüllt, die Genehmigung weiterhin nicht mit der Begründung verweigern darf, dass die Leistung für ihn fachfremd ist.

- sich die Fachgebietsgrenzen für einen Vertragsarzt aus der für sein Gebiet aktuell gültigen Weiterbildungsordnung ergeben - unabhängig davon, nach welcher Weiterbildungsordnung er die Berechtigung zum Führen seiner Facharztbezeichnung erworben hat (BSG, Urt. v. 4. 5.2016 - B 6 KA 13/15 R -, MedR 2017, S. 179 ff. mit Anm.).

Eingesandt und bearbeitet von

Rechtsanwalt Dr. iur. Herbert Schiller,

Römerweg 5a, 86199 Augsburg, Deutschland 